\title{
Das Ende des Politischen? Demokratische Politik und Künstliche Intelligenz
}

\author{
Ann-Kathrin Koster
}

Angenommen: 12. Juli 2021 / Online publiziert: 9. August 2021

(C) Der/die Autor(en) 2021

Zusammenfassung In jüngster Zeit findet innerhalb der politiktheoretischen Forschung eine vermehrte Auseinandersetzung mit algorithmenbasierten Systemen statt. Diese ist geprägt von der Behauptung einer neuen algorithmischen Regierungsweise, die aufgrund ihrer reduktionistisch-formalen Logik sowohl plurale Sinnzusammenhänge untergräbt als auch die individuelle Entfaltung reflexiver Urteilsbildung unterminiert. Entgegen dieser Annahmen argumentiert der vorliegende Beitrag, dass der Einsatz dieser digitalen Technologien im politischen Kontext nicht zwangsläufig in eine post-politische Verfasstheit von Gesellschaft münden muss. Algorithmische Systeme lassen sich als spezifische epistemische Verfahren verstehen, deren operativer Gebrauch symbolischer Inputs zwar einer schließenden, ontologisierenden Logik folgt und für sich genommen kontingenzreduzierende und latent anti-politische Wirkungen zeitigt. Demokratische Gesellschaften zeichnen sich aber hinsichtlich ihrer kontingenztheoretischen Verfahren gerade dadurch aus, dass ihre befragende Logik eine Inkorporation solcher Ontologisierungen ermöglicht. Es geht dann vielmehr darum, die Bedingungen ihrer Politisierung in den Blick zu nehmen.

Schlüsselwörter Algorithmen · Kontingenz · Politisches Handeln · Pluralität · Rationalisierung

\footnotetext{
Ann-Kathrin Koster $(\square)$

Philosophische Fakultät, Schaufler Lab, Technische Universität Dresden, 01067 Dresden, Deutschland

E-Mail: Ann-Kathrin.Koster@mailbox.TU-Dresden.de
} 


\title{
The end of the political? Democratic politics and artificial intelligence
}

\begin{abstract}
Over the last years, political theory has engaged increasingly in a discussion on algorithm-based systems and its socio-political implications. The debate on this issue is characterized by the assertion of a new algorithmic mode of governance that, due to its deterministic and formal logic, both undermines plural contexts of meaning and the individual unfolding of reflexive judgment. Contrary to these assumptions, this article argues that the use of such digital technologies in a political context does not necessarily result in a post-political society. Algorithm-based systems can be understood as specific epistemic procedures whose operative use of its symbolic input follows a conclusive, ontologizing logic and in itself has a contingency-reducing and latently anti-political effect. Democratic societies, however, are characterized with regard to their contingency-theoretical procedures precisely by the fact that their critical logic enables an incorporation of such ontologizations. Based on this, it seems rather to be about the conditions of their politicization within a democratic context.
\end{abstract}

Keywords Algorithms · Contingency $\cdot$ Political action $\cdot$ Pluralism $\cdot$ Rationalization

\section{Einleitung}

Algorithmischen Systemen wird in geistes- und sozialwissenschaftlichen Disziplinen zunehmend mit Interesse begegnet. Dabei erhalten Algorithmen-basierte Lernverfahren unter dem Sammelbegriff „Künstliche Intelligenz“ (KI) besondere Aufmerksamkeit. Aus einer politiktheoretischen Perspektive dient das Kriterium freiheitlicher Selbstregierung bisher als wichtiger Anknüpfungspunkt der normativ-kritischen Auseinandersetzung, unter dem dann relationale Verschiebungen zwischen Mensch und Maschine zu Lasten der Bürger*innen konstatiert und entsprechend kritisch reflektiert werden. Dahinter steht die Annahme, dass die Logik demokratischer Ordnungen gefährdet ist, da sich durch den Einsatz algorithmischer Systeme neue soziale wie politische Steuerungslogiken - Stichwort algorithmic governance durchsetzen, die insbesondere Formen individuell-freiheitlicher Entfaltung und reflektierter Urteilsbildung unterlaufen. Der vorliegende Beitrag widmet sich diesem Wechselverhältnis zwischen technologischer und demokratischer Rationalität und bearbeitet die Frage, inwiefern die Funktionslogik algorithmischer Systeme mit den kontingenzorientierten Rationalitäten demokratischer Politik in Konflikt gerät. Der Beitrag argumentiert, dass Verfahren Künstlicher Intelligenz zwar grundsätzlich an einer ontologischen Schwelle und innerhalb einer reduktionistischen Epistemologie operieren, aber nicht zwangsläufig in eine post-politische Gesellschaft münden. Dies deshalb, da demokratische Gesellschaften sich hinsichtlich ihrer epistemologischen Prinzipien grundsätzlich dadurch auszeichnen, dass ihre befragende Logik eine Inkorporation solcher systemimmanenten Rationalitäten ermöglicht.

Das Argument wird in vier Schritten entfaltet. Der Beitrag geht in einem ersten Schritt auf die bisherige politiktheoretische Diskussion zur Kritik an algorithmischen Verfahren und KI ein und verdeutlicht, dass diese sich durch eine Kritik instrumentel- 
ler Vernunft sowie eine technikdeterministische Gefährdung der mündigen Bürger*in auszeichnet (Kap. 2). Daran anknüpfend wird die in der Diskussion angerissene Verfahrenslogik von KI in einem breiteren ideengeschichtlichen Kontext verortet. Ziel dieser Rückbindung ist es, die numerisch-rationalistische Logik digitaler Technologien in den Kontext eines spezifisch technischen Modus des Symbolgebrauchs zu setzen. Dadurch wird die mit Algorithmen einhergehende Ontologisierung über eine starre Bindung an konkrete mathematische Verfahrensregeln ersichtlich (Kap. 3). So ergibt sich dann das Bild einer rationalen und berechnenden Verfahrenslogik der Maschine. Auf den ersten Blick stellt dies, wie in der bisherigen politikwissenschaftlichen Diskussion dargelegt, ein zentrales Problem für pluralistisch verfasste Gesellschaften dar. Dagegen setzt der Beitrag die Ansicht, dass jenseits einer Reduktion auf das Bild einer berechnenden Verfahrenslogik der Maschine algorithmische Systeme jedoch in ihrer gesellschaftlichen Einbettung betrachtet werden müssen. Diese können, wenn es um die Beurteilung ihre sozio-politischen Auswirkungen geht, nicht ausschließlich auf die maschinelle Verfahrenslogik reduziert werden. Unter Berücksichtigung techniksoziologischer wie -philosophischer Ansätze erfolgt daher eine Kritik am starren Mensch-Maschine-Verhältnis und der daraus abgeleiteten Schlussfolgerung eines individuellen Freiheitsverlusts (Kap. 4). Stattdessen plädiert der Beitrag für ein zwar spannungsgeladenes, aber offenes Aushandlungsverhältnis zwischen Gesellschaft und Technik, das sich als demokratietheoretisch anschlussfähiger erweist. Unter Bezugnahme auf radikaldemokratische Theorieansätze rückt der Beitrag den Fokus auf die Annahme, dass demokratische Verfahren sich insbesondere durch einen ergebnisoffenen, mediatisierten Umgang mit epistemischen Konflikten auszeichnen - etwa indem die spezifischen Rationalitäten von Verfahren oder Prozessen ausgestellt und kritisch hinterfragt werden. Demokratische Verfahren stellen sich somit als de-ontologisierende Praktiken dar (Kap. 5). Es geht also darum, ein kontingentes Spannungsverhältnis zwischen Verfahren Künstlicher Intelligenz und demokratischer Mediation zu betonen, ohne dabei die Verfahrensweise algorithmischer Systeme mit ihren gesellschaftspolitischen Auswirkungen gleichzusetzen und dadurch eine Totalität algorithmischer Logiken anzunehmen. Demokratische Ordnungen als ein spezifisches auf Vermittlung und Befragung hin ausgerichtetes Verhältnis zu definieren, welches stets zwischen unterschiedlichen Rationalitäten und mit ihnen verbundenen technologisch-institutionellen Arrangements dynamisch reagiert, ermöglicht dann auch einen gezielten Umgang mit technologischen Systemen, wie sie KI-Verfahren darstellen, anzunehmen. Dies zeigen etwa in jüngster Zeit Proteste gegen den zunehmenden Einsatz algorithmischer Verfahren.

\section{Algorithmic Governance. Eine politiktheoretische Auseinandersetzung}

Algorithmen, maschinelles Lernen, autonome Entscheidungsfindung, Künstliche Intelligenz - all diese Begriffe werden zunehmend zu Gegenständen politikwissenschaftlicher Forschung (Hofmann et al. 2019; König 2018, 2019; Müller-Mall 2020; Sudmann 2019; Thiel 2020; Ulbricht 2020; Unger und Ungern-Sternberger 2019). Sie weisen deutliche Überschneidungen auf, setzen allerdings unterschiedliche 
Schwerpunkte und knüpfen an unterschiedliche Forschungsstränge an. So setzen sich etwa die „Critical Algorithm Studies“ kritisch mit dem Einsatz von Algorithmen und den damit verbundenen gesellschaftlichen Auswirkungen auseinander (Kitchin 2017). Der Begriff der Algorithmisierung findet prominenten Niederschlag in der soziologischen Forschung (Ulbricht 2020) und der Begriff des maschinellen Lernens wird eng an Fortschritte bei der Entwicklung Künstlicher Intelligenz genknüpft (Sudmann 2018). In allen Fällen geht es jedoch stets um den Einfluss von oder das Wechselverhältnis zwischen Technik und Gesellschaft bzw. Politik, wobei insbesondere die den Systemen inhärenten Algorithmen in den Mittelpunkt gerückt werden.

Algorithmen verweisen zunächst auf eine spezifische Technisierung, die sich als eine formale mathematische Anweisungsfolge darstellt (Müller-Mall 2020, S. 11). Sie dienen als Infrastruktur zwischen unterschiedlichen technologischen und nichttechnologischen Einheiten und nehmen dadurch Einfluss auf die Konstitution gesellschaftlicher Ordnungen (Seyfert und Roberge 2017; Ulbricht 2020). Diese Wirkung wird häufig unter Begriffen wie algorithmic governance, algorithmic governmentality oder algorithmischer Regulierung gefasst (Just und Latzer 2017; Rouvroy 2011; Yeung 2018). Auf Basis großer Datenmengen treffen algorithmenbasierte Systeme Entscheidungen mit politischem Bezug, etwa wenn es um die Strukturierung sozialer wie politischer Interaktionen geht (Just und Latzer 2017; König 2019; Rouvroy 2011; siehe für einen umfassenden Überblick: Katzenbach und Ulbricht 2019). In den Mittelpunkt rückt die Annahme, dass Entscheidungen nun nicht mehr aktiv getroffen werden, sondern in (maschinelle) Verfahren überführt und so technokratisch, das heißt insbesondere auf Grundlage statistischer Häufung oder Ähnlichkeit, aufgelöst werden. Aus politischer Perspektive bedeutet eine solche technische Übertragung dann, dass politische Fragen nicht mehr nach der ihnen inhärenten Logik entschieden werden, sondern ausschließlich verfahrenslogisch behandelt werden: Akte der Verwaltung ersetzen (politisches) Handeln (Müller-Mall 2020, S. 32 f.).

Das betont ebenfalls der Begriff der algorithmischen Entscheidungsfindung (,algorithmic decision making"). Er hebt in diesem Kontext hervor, dass algorithmische Systeme nicht nur große Datenmengen auswerten und analysieren, sondern auf Basis eben jener Analysen auch zunehmend weitreichende Entscheidungen treffen (AlgorithmWatch 2020). Aus dieser delegierten und automatisierten Entscheidungskompetenz folgt dann vermehrt eine Zuschreibung von Selbstständigkeit, weshalb auch von automated decision making (ADM) gesprochen werden kann: „ADM can be conceptualized as instances in which algorithms or an AI are used to collect, process, models, and use data to make automated decisions. In turn, feedback from these decisions is then used by the system to improve itself." (Araujo et al. 2020, S. 612) Konkrete Entscheidungen werden von Algorithmen (mit-)vorbereitet, von ihnen durchgeführt oder gar endgültig getroffen. Grundlegende soziale wie politische Prozesse, die zuvor von menschlicher Hand vollzogen wurden, werden demnach zunehmend an Maschinen delegiert und von diesen nach spezifischen Vorgaberegeln verrichtet. Ein häufig gewähltes Beispiel im politikwissenschaftlichen Kontext ist die Einschränkung des Zugangs zu unterschiedlich gelagerten Informationen im Bereich sozialer Medien und des Nachrichtenwesens. Hier, so eine gebräuchliche Argumentation, verdrängen Algorithmen die zuvor dominanten nachrichtlichen In- 
termediäre und üben somit Einfluss auf politische Entscheidungen und Urteile von Individuen aus, indem sie die wahrzunehmenden Informationen individualisieren, anstatt diese zu pluralisieren und an eine allgemeinere Leser*innenschaft zu binden (Gillespie 2018; Ungern-Sternberger 2019). Aber auch in weiteren Bereichen finden solche Anwendungen Einsatz, so etwa im Bereich der Polizeiarbeit. Unter dem Begriff des „Predictive Policing“ kommt hier eine Prognosesoftware zum Einsatz, die auf Basis vernetzter Polizeidatenbanken und statistischer Verfahren die Wahrscheinlichkeiten von Straftaten berechnet. Ziel des Einsatzes ist es, etwa im Bereich des Wohnungseinbruchs, die Polizeiarbeit zu optimieren (Egbert 2020, S. 78 f.; für einen allgemeinen Überblick über Prognoseverfahren siehe Lopez 2021).

Trotz der unterschiedlichen Schwerpunktsetzungen werden die hier angeführten Begriffe und Verfahren häufig unter dem Terminus der Künstlichen Intelligenz subsumiert. Diese erscheint damit zunehmend als eine Art Universaltechnologie, weil sie für immer mehr nicht-technische Probleme als (Teil-)Lösung suggeriert wird (Sudmann 2018, S. $10 \mathrm{ff}$.). KI tritt mit Wolfgang Ertel gesprochen als eine Art „Werkstatt mit einer überschaubaren Anzahl an Werkzeugen“ (Ertel 2016, S. 12) auf, die anhand von verschiedenen, aber begrenzten Ansätzen und Lösungswegen einer Vielzahl an technischen, wie auch gesellschaftlichen Problemen begegnen kann (konkreter zu den einzelnen Lösungswegen siehe Domingos 2015). Es ist genau jener Themenkomplex, dem sich die geistes- wie sozialwissenschaftliche Forschung annimmt und in ihren Analysen darauf verweist, dass die damit einhergehende Algorithmisierung, also das umfassende Eindringen algorithmischer Systeme in die Lebenswelt, politische Logiken beeinflusst oder gar zu überschreiben droht. Somit setzt sich in mancherlei Hinsicht die These von der Kolonisierung der Lebenswelt an dieser Stelle unter Bezugnahme auf das Digitale fort (Houben 2018). Denn unter den Bedingungen algorithmischer Ordnungsbildung seien „Interaktionen und Erfahrungen“ nun von der „Entfaltung von Rechenleistungen abhängig“ (Roberge und Seyfert 2017, S. 7) bzw. träten in ein enges Wechselverhältnis zu diesen. Die Kolonisierung wird hierbei eng an die algorithmische Rechenleistung und eine damit verbundene spezifische Logik geknüpft. Diese zeichne sich, so Becker und Seubert, als eine numerischen Rationalität aus, die gerade nicht die „Mannigfaltigkeit und Ambiguität weltlicher und sozialer Phänomene [anerkennt, sondern diese] auf einen Zahlenwert eindampft und daraus soziale Strukturen und Kausalitäten ableitet." (Becker und Seubert 2020, S. 238) Es geht also in allen Fällen um Vereinheitlichung, wodurch es zu einer Vereinseitigung von Erkenntnisformen komme, die eine Komplexitätsreduktion bedinge (247). Eine solche Komplexitätsreduktion stellt sich als Gegenspielerin einer demokratischen Pluralitätsannahme dar, die darauf verweist, dass stets mannigfaltige Logiken existierten, wobei gerade unterschiedliche und voneinander abweichende Deutungsangebote das politische Miteinander bestimmen. Statt lebensweltlicher Pluralität werden durch den vermehrten Einsatz von Algorithmen-basierten Verfahren numerische Parameter sowie die innerhalb der Mustererkennung an Bedeutung gewinnenden Kenngrößen ,Wiederholung, Serialität, Symmetrie“ (Mersch 2019, S. 852) zu zentralen Gradmessern politischer Organisation.

Bei einer Betrachtung der politiktheoretischen Analysen fällt auf, dass algorithmischen Verfahren, insbesondere der sich darin ausbreitenden rationalistischen Logik, 
das Potenzial zur umfassenden Determination politischer Prozesse zugesprochen wird, die von Ambivalenzen, Kontextfaktoren und Brüchen stark abstrahiert. Die Annahme einer dualistischen Spannung zwischen numerisch-reduktionistischer und pluralistisch-reflexiver Logik bildet den schematischen Hintergrund dieses Arguments. Deutlich wird dies etwa, wenn der Modus algorithmischer Governance - also die gesellschaftliche Steuerung durch Algorithmen - als ,grundsätzlich der Idee der Selbstregierung der Bürger entgegen [stehend]“ (König 2018, S. 303) stilisiert wird. Damit werden Algorithmen nicht als in gesellschaftliche Aushandlungsprozesse eingebettet verstanden, sondern vielmehr als externe Wirkkraft beschrieben, welche sich gesellschaftlich wie politisch nicht einfangen lässt. Dies korreliert zugleich mit zwei Regulierungsparadigmen, einem technischen und einem sozialen. Wird Ersteres als besonders effektiv, machtvoll und auch leistungsstark definiert, führt dies meist zwangsläufig zur Schwächung bzw. Unterminierung des Letzteren (Katzenbach und Ulbricht 2019, S. 2f.). Statt gerade ein Moment der interaktiven Aushandlung in den Blick zu nehmen, wird das Aufeinandertreffen in ein Konkurrenzverhältnis aufgelöst. Damit bleibt die Analyse des konkreten Aufeinandertreffens der beiden Logiken hinter einem postulierten Dominanzgewinn der technischen Logik zurück. ${ }^{1}$

Im Wechselverhältnis zwischen Individuum bzw. Gesellschaft und Algorithmenbasierten Verfahren wird insgesamt eine Situation der Rivalität angenommen, und zwar ganz besonders dann, wenn es um die Steuerung individuellen Verhaltens geht, welches vordergründig ins Visier eines solchen Diskurses gerät. Die Steuerungsfunktion von Algorithmen, etwa in ihrer Form als plattformbasierte Kommunikationsmacht, führe somit zu einer Rationalitätsverschiebung zu Lasten demokratischer Systeme, da sie selbst, so Ungern-Sternberger, den Newsfeed als digital konstitutiven Ort der demokratischen Meinungsbildung sortierten und strukturierten (2019, S. 10) oder im Bereich der Produktplatzierung gezielt die individuellen Bedürfnisse erkundeten und anschließend über Werbung bedienten. In kritischen Diagnosen wird eine algorithmische Steuerung genau dort zum Problem, wo ausgehend von algorithmischen Systemen eine individualistisch-freiheitliche Logik der Bedürfnisbefriedigung die kollektiv-reflexive Freiheit mündiger Bürger*innen überschreibt, die jedoch für demokratische Ordnungen zentral ist (König 2019, S. 443; 452). Auch nach Becker und Seubert übernimmt die digitale Logik zunehmend die Steuerung des privaten Lebensbereichs. Ausschlaggebend werde in diesem Kontext die algorithmische Rationalität, die die ,Selbst-und Fremdwahrnehmung privater Individuen“ einer „,numerischen, metrischen Selbsterfassung [unterwerfe], die auf einer Idee vermeintlich objektiver, weil datenbasierter Selbstbestimmung“ (2020, S. 250) aufbaue. Algorithmen-basierte digitale Technologien in Gestalt einer numerisch angeleiteten Erkenntnisform werden somit auf unterschiedlichen Ebenen zum Hindernis eines selbstbestimmten und darüber hinaus pluralistisch definierten Lebens und damit folglich auch des demokratischen Miteinanders, welches im Kern als pluralistisch definiert wird. Zusammengefasst lässt sich daher feststellen, dass die politiktheoretische Auseinandersetzung mit der Einbettung von KI in politische

\footnotetext{
${ }^{1}$ Zum Vergleich einer Kritik an einer reduktionistischen Perspektive auf das Mensch-Maschine-Verhältnis, allerdings mit ökonomischem Bezugspunkt, siehe Mackenzie (2017, S. 16-18).
} 
und gesellschaftliche Zusammenhänge stark von schematischen und bisweilen technikdeterministischen Perspektiven dominiert wird.

Die Fokussierung auf die numerische Form Algorithmen-basierter Verfahren lässt sich dabei historisch betrachtet insbesondere an die frühe KI-Entwicklung rückbinden. Logische Schlussfolgerungsregeln als wesentlicher Baustein von KI-Verfahren dominierten insbesondere die 1950er und 1960er-Jahre des Entwicklungsdiskurses der KI-Forschung. Es scheint demnach auf den ersten Blick sinnvoll, sich auf die konkreten technischen Verfahren und ihre Beschaffenheit in der Auseinandersetzung mit Algorithmen-basierten Technologien zu konzentrieren und diese als wesentlichen Anknüpfungspunkt einer politikwissenschaftlichen Beurteilung heranzuziehen. Ein Blick in die KI-Geschichte macht jedoch schnell deutlich, dass die Entwicklung von KI nicht bloß von mathematischen Fragen und numerischen Parametern geprägt ist, sondern davon, wie sich soziale und individuelle Verhaltensweisen technisch ab- und nachbilden lassen. Denn KI-Verfahren wurden in enge Anlehnung an den Menschen - insbesondere menschliche mentale Prozesse - als Gegenüber und Vergleichsfolie der Technik konstruiert. Dabei wird deutlich, dass KI-Verfahren zwar grundlegend von einer numerischen Rationalität geprägt sind, diese jedoch in stetigem Austausch und damit in Abhängigkeit und Wechselwirkung mit ihrer Umwelt stehen. Gerade diese Einbettung der Technik selbst verweist dabei auf eine von Brüchen und Ambivalenzen geprägten Mensch-Maschine-Beziehung, die wiederum eine zu den bisherigen Deutungen kontrastierende Lesart ermöglicht, welche gerade statt der Verfahrenslogik die Aushandlungsprozesse zwischen verschiedenen soziotechnischen Entitäten in den Blick rückt.

\section{Die sequentielle Natur des Denkens. Leitideen Künstlicher Intelligenz-Forschung}

Die Betonung numerischer Rationalitäten, wie wir sie in der politikwissenschaftlichen Auseinandersetzung finden, weisen vielfach Ähnlichkeiten mit einem insbesondere die frühe KI-Forschung der 1950er-Jahre dominierenden Paradigma auf: dem der symbolischen KI (Ernst et al. 2019, S. 11). Symbolische KI-Ansätze orientieren sich in der Modellierung algorithmischer Systeme an konkreten logikbasierten Schlussfolgerungsregeln, welche mathematisch über Kalküle, also spezifische Anwendungsregeln, modelliert werden, sodass konkrete Sachverhalte über mathematische Berechnungen handhabbar und zugänglich gemacht werden können (Domingos 2015, S. 52; Ernst et al. 2019, S. 11). Was KI-Verfahren ausführen, geschieht demnach auf der Basis sequentieller Operationen, die jeweils individuelle Rechenschritte darstellen und zu einem konkreten Ergebnis bzw. einer Zuordnung führen - so die prinzipielle Vorgehensweise von symbolischen Ansätzen. Die Betonung der numerischen Rationalität, wie sie im vorigen Abschnitt ausgearbeitet wurde, ist also nicht neu. Bereits in der frühen Genese Künstlicher Intelligenz wurde auf die sie anleitende rationalistische Tradition verwiesen und als wesentliches Leitbild in der Konstruktion intelligenter Maschinen herausgestellt (Winograd und Flores 1989; gegenwärtig siehe insbesondere Russell und Norvig 2012, die KI als eine rationale Agentin definieren). Ein genauerer Blick offenbart die frühen epistemischen Weichenstellungen 
des maschinellen Verfahrens und verdeutlicht, dass ihre rationalistische Verankerung tiefer geht, als es die gegenwärtig angeführten mathematischen Termini der Statistik und Wahrscheinlichkeit nahelegen, bedingt die technische Modellierung von konkreten Lernverfahren doch immer auch eine spezifisch mechanische Form der Wissensgenerierung (Domingos 2015, S. 52; Ernst et al. 2019, S. 10 ff.; Hörl et al. 2021a, S. 9). KI-Forschung stand vor allem in ihren Anfangsjahren verstärkt in der Tradition der Modellierung mentaler Funktionen des Menschen - die frühe KIForschung drehte sich um die mechanische Modellierung des Denkens schlechthin, wobei es insbesondere darum ging, die zentralen Parameter des Denkens systematisch zu erfassen (Boden 1990, S. 2 f.; McCarthy et al. 1955, S. 2 f.).

Grundsätzlich orientieren sich KI-Verfahren, die sich unter dem symbolischen Paradigma subsummieren lassen, an philosophischen Überlegungen, wonach der Mensch Zugang zur Welt über Symbole, also sinnbildliche Repräsentationen, findet, indem er diese entsprechend verschiedener Sachverhalte manipuliert und so in Kommunikation mit der ihn umgebenden Welt tritt. Prominent für eine solche Sichtweise steht Alan Turing, welcher den Prozess des Berechnens als Umformung von Symbolen definierte und diese als von Maschinen ausführbar verstand, da er die Manipulation von Symbolen mit der Lösung mathematischer Gleichungen gleichsetzte (Turing 1990; Boden 1990). Symbolmanipulationen maschinell auszuführen, setzte im Rahmen der mechanischen Modellierung also auf der Ebene der Regeln an, die jene Erstellung und Veränderung sinnbildlicher Repräsentationen anleiten und in konkrete Abfolgen von Berechnungen überführt werden können. KI-Forschung unter dem symbolischen Ansatz lässt sich demnach ganz konkret als Feld der Erkundung der Interaktion des Menschen mit der sich ihm darstellenden Umwelt und ihrer mechanischen Modellierung verstehen: wie er sie wahrnimmt, verarbeitet und zu einer kohärenten Interpretation dessen gelangt, was sich ihm entgegenstellt und wie diese Gegenüberstellung in konkreten Regeln des Schlussfolgerns festgehalten und übertragen werden kann. KI-Verfahren erscheinen so als maschinelle Erkenntnisform, die es gilt, selbst in den Blick zu nehmen (Winograd und Flores 1989, S. 25; Coy 1993, S. 31).

\subsection{Erkenntnis als Resultat regelgeleiteten Denkens}

Der Analogieschluss zwischen menschlichem Geist und Maschine bezog sich in der frühen KI-Forschung im Besonderen auf das zur Erkenntnis fähige Subjekt (Hörl et al. 2021a, S. 9f.). Zum Bezugspunkt der Modellierung wurde die gezielte Erfassung weltlicher Verhältnisse durch das Individuum, insbesondere die sich darin manifestierende Fähigkeit zur Analyse eben jener Weltverhältnisse. Bedeutungsproduktion wurde hier weniger im Prozess der Interaktion verortet, als ausschließlich inner-individuell definiert. Zum Leitbild der KI-Forschung avancierte so eine Definition von Erkenntnis als Produkt des „Umgang[s] mit dem Wissen von einer objektiven Welt" (Winograd und Flores 1989, S. 32; Glymour et al. 2006, S. 7 f.), welche dem Menschen in einer objektiven Art und Weise zugänglich sei. Wird die Welt als objektiv erfassbar verstanden, so muss lediglich nach konkreten Regeln und Schemata ihrer systematischen Erfassung gesucht werden. Denken wurde somit enggeführt auf einen logischen Vorgang, vor allem auf „,norms of good reasoning“ 
(Isaac 2019, S. 13f.): Denken erschöpft sich hier im Führen von Beweisen, die selbst logik-spezifischen Regeln folgen. Frei nach dem Motto, dass die Welt in einer Fülle von Tatsachen aufgeht, die wiederum in eine „Reihe von ,Elementarsätzen““ (Mersch 2019, S. 110) zu gliedern ist, stellt sich die Erzeugung von Wissen als ein Akt des logischen Schlussfolgerns dar (Haugeland 1993, S. 23 ff.), woran sich letztlich vor allem auch die mechanische Modellierung abarbeitet.

Zentral wird somit der Prozess des mathematischen Schlussfolgerns als Informationsverarbeitung in einem logischen Sinne. Leiten Regeln das logisch-beweisführende Denken an, so findet sich hier eine Brücke zu maschinellen Rechenverfahren. ${ }^{2}$ Auf erkenntnistheoretischer Ebene der Maschine setzt sich eine Sichtweise durch, die Denkprozesse eng an die Befolgung spezifischer regelgeleiteter Operationen knüpft. Denken geht demnach also in Prozessen des logischen Schlussfolgerns vollständig auf (McCarthy 2006, S. 7; Winograd und Flores 1989, S. 39). Große Teile der KI-Forschung drehten sich daher um die Frage der technischen Ausgestaltung des Problemlösungsverfahrens. Es sind die Regeln des formalen Systems, welche zum Rahmen der Wirklichkeit und damit ausschlaggebend für ein gefestigtes Bild der Realität werden (ausführlich dazu Haugeland 1993). Um diese Regeln maschinell zu erfassen, wird eine ,universelle Kalkülsprache“ (Bächle 2016, S. 22) für die Funktionalität von intelligenten Maschinen essentiell. Kalküle, so nahm man an, erlaubten Maschinen, aus einer begrenzten Anzahl an Inputs eine unbegrenzte Anzahl an Outputs zu produzieren und somit abgeleitet auch die Produktion immer neuer Erkenntnisse anhand spezifischer logischer Schlussfolgerungssätze, da Kalküle als konkrete Anwendungsregeln universell auf die unterschiedlichsten Bereiche angewendet werden können und dennoch in der Lage sind, konkretes Wissen zu erzeugen (Bächle 2016, S. 22; Krämer 1988, S. 59).

Eine solche Fokussierung auf mathematische Operationsregeln birgt jedoch bereits auf maschineller Ebene eine performative Schließung: Zum zentralen Referenzpunkt wird nicht mehr der situative Kontext des Inputs, seine Besonderheit oder Spezifität, sondern die Korrektheit der logischen Operationen selbst, also die richtige Anwendung und Umsetzung konkreter Rechen- und Lösungswege. Dabei werden die zu verarbeitenden Sachverhalte aus ihren unterschiedlichen Kontexten herausgelöst und als isolierte Phänomene betrachtet, wobei die pluralen Deutungsmöglichkeiten durch die Kohärenz des Regelsystems ersetzt werden. Die Wahrheit und Anerkennungswürdigkeit eines Sachverhalts speist sich nunmehr aus der Kohärenz des Systems selbst, nicht aus einer spezifisch weltlichen Verortung oder einem konkreten Aushandlungsprozess (Bächle 2016, S. 22). Im Zuge der Bindung an eine Formalsprache spricht Sabine Müller-Mall mit Blick auf den gegenwärtigen Einsatz von algorithmischen Systemen von einer ,,Verengung der Interpretationsmöglichkeiten“" (2020, S. 67) in Bezug auf konkrete weltliche Sachverhalte.

\footnotetext{
${ }^{2}$ Es war Thomas Hobbes, der ideengeschichtlich die enge Beziehung zwischen Denken und Berechnen besonders früh betonte (Haugeland 1993; Isaac 2019; Krämer 1988).
} 


\subsection{Die maschinelle Disposition zur Ontologisierung}

Es waren insbesondere Sybille Krämer und Bettina Heintz, die bereits sehr früh in der Auseinandersetzung mit Computern von einer viel grundlegenderen Veränderung der Beziehung zwischen maschineller Verarbeitung und weltlichen Bezugsobjekten sprachen, die sich im Zuge der Einführung formaler Systeme ereigneten und sich nicht im Begriff einer Rationalisierung qua Technik erschöpfen (Krämer 1988; Heintz 1993). Denn wenn als Referenzpunkt der zu verarbeitenden Informationseinheit nicht mehr die weltlichen Verhältnisse dienen, sondern eine enge Kopplung an die Berechnungsvorschriften erfolgt, dann übt dies Einfluss auf die Bedeutungsproduktion, also die Entstehung von Sinn selbst, aus (Krämer 1991, S. 4). Wie in Krämers bzw. Heintz' Fall können neben Computern ebenso KI-Verfahren als Formalsysteme verstanden werden und verweisen somit auf eine sich ändernde Sinnproduktion. Diese veränderte Sinnproduktion ergibt sich Krämer zufolge aus einem Bruch im Verhältnis zwischen Symbol und Symbolisiertem, also dem Zeichen und dem, auf das das Zeichen als solches verweist. Nach Sybille Krämer kommt es in formalen Systemen zu einer Umkehr des Verweisungszusammenhangs zwischen Symbol und Symbolisiertem, der in einem Bruch zwischen beiden begründet liegt.

Krämer argumentiert an dieser Stelle, dass, so lange „die Existenz des Symbols verankert wird in der Existenz des Symbolisierten, welche dem Symbol ontologisch vorgeordnet wird, solange bleibt auch ein formaler Gebrauch von Symbolen ausgeschlossen. Denn ,Formalität' hat die ,Emanzipation“ der Zeichen gegenüber dem was in ihnen zur Darstellung gelangt, zur Voraussetzung, kann anders doch die Idee, Symbole in Kalkülen zu organisieren, die zuerst aufgebaut und dann erst interpretiert werden können, nicht gefaßt werden“ (Krämer 1991, S. 11). Werden Symbole in Kalkülen organisiert und mit ihnen operiert, geschieht dies letztlich zum Preis der Loslösung von ihren pluralen Bedeutungsebenen.

Formale Systeme ändern das Verhältnis zwischen Symbol und Symbolisiertem, insofern sich die Umformung der Symbole von den ihnen inhärenten Bedeutungsmöglichkeiten entkoppeln und ausschließlich an die den Kalkülen inhärenten Operationsregeln koppeln. Es sind also ,nicht mehr die Dinge[, die] den Zeichen ihre Bedeutung verleihen, vielmehr [sind es] die Zeichen[, die] die Dinge als epistemische Gegenstände erst konstituieren“ (Krämer 1991, S. 4f.; Bächle 2016) - dies jedoch in einer ausschließlich operativen Art und Weise, welche die Regeln des logischen Schlussfolgerns vorgeben und somit stets gleichförmig verlaufen. Es ist somit unerheblich, ob mit Hilfe formaler Systeme Spam-Emails gefiltert oder Nachrichten sortiert werden. Zu der Operation mit Ziffern, also der numerischen Ausgestaltung solcher Verfahren, tritt auf der ontologischen Ebene eine operative Schließung. Die Maschine selbst scheint nur noch weit entfernt etwas mit der sie umgebenden Welt und der sich ihr über die Informationen darstellenden Wirklichkeit zu tun zu haben. Vielmehr werden die Inputs zum Spielball des Regelsystems selbst, das sich mit jeder erneuten Eingabe selbst prüfen und bestätigen kann. Formalistische Verfahren, wie sie auch KI-Verfahren darstellen, produzieren Aussagen über die Welt in einem Prozess der ausschließlich ,immanent definiert“ ist und so gerade ontologisierende Wirkung zeitigt (Heintz 1993, S. 27; siehe auch Bächle 2016, S. 22). 


\subsection{Künstliche Intelligenz in vernetzten Umgebungen des 21. Jahrhunderts}

Eine regelgebundene inner-maschinelle Bestimmung von Wirklichkeitsverhältnissen wird gerade dann zum Problem, wenn die intelligente Maschine aufhört, ein isolierter Apparat zu sein. Gegenwärtig sind solche Verfahren vor allem in über Daten vernetzte Umgebungen eingebettet. Prominente Beispiele sind hier etwa das Smart Home, autonomes Fahren, das Internet der Dinge wie ebenso die Interfaces sozialer Medien (Sprenger 2019). Aber auch außerhalb dieser Beispiele sind KI-Verfahren, etwa über die alltägliche Nutzung des Smartphones, in hohem Maße mit dem Menschen wie auch der menschlichen Lebenswelt relational verschränkt. Während die Ontologisierung bisher vor allem als eine maschinelle Disposition herausgestellt wurde, wird dies vor allem in einer kontextuellen Einbettung zum Problem. Denn anders als die reine Auseinandersetzung mit der historischen Entwicklung von KI-Verfahren nahelegt, wird zunehmend deutlich, dass KI-Verfahren als Mitspieler*innen betrachtet werden müssen, die „,das gesellschaftliche Selbstbewusstsein (mit)[...] gestalten“ (Ulbricht et al. 2018, S. 155) und das vor allem in einer aktiven und auch eigenwilligen Art und Weise. Die regelgeleitete Erzeugung von Wirklichkeit durch KIVerfahren erscheint gerade im Wechselspiel mit umfangreichen Datensätzen in aller Deutlichkeit in ihrer ,ontologisch-performative[n]“ Dimension (Ulbricht et al. 2018, S. 156; Amoore 2020; Bucher 2018), welche in einem engen Wechselverhältnis zum „Prozess der gesellschaftlichen Selbsterfassung" steht und so zu einem aktiven Teil der „gesellschaftliche[n] Sinnproduktion“ erwächst (Berg et al. 2020, S. 176). Ihre ontologisierenden Effekte finden also gerade in Momenten der Interaktion und nicht der externen Einwirkung Eingang in gesellschaftliche Prozesse: So sind gegenwärtige algorithmische Systeme in ihrer Verfahrensweise als „ontological politics“ zu verstehen, die „drifting into the ways in which people think and talk about everything from the economy to knowledge production to culture." (Bucher 2018, S. 39)

Es ist also nicht eine technik-immanente Produktion von Wahrheit, die zum Problem wird, sondern das Ineinanderwirken unterschiedlicher Systeme und den ihnen inhärenten Logiken, welche zum Aufkommen vielfach diagnostizierter pathologischer Folgen führt (Müller-Mall 2020, S. 67). So entsteht eine komplexere Problemlage, in der gesellschaftliche Risiken aus Mensch-Maschine-Interaktionen und den konkreten Interdependenzen entstehen und nicht einfach monokausal aus den Logiken der Maschine abgeleitet werden können. KI muss somit als Komplex definiert werden, der aktiv die Beziehung zwischen Individuum und Kollektiv beeinflusst, indem ,,die Realitäten, [welche] von Gesellschaft, Politik, Öffentlichkeit und Bürgerinnen und Bürgern kollektiv gelebt werden“ (Ulbricht et al. 2018, S. 162; Mühlhoff 2020), performativ mit erzeugt werden, wobei KI-Verfahren die ihnen zugrunde liegende rationale Sichtweise aktiv in Prozesse der Sinnproduktion einspeisen, dabei jedoch zwangsläufig auf weitere Rationalitäten treffen (Amoore 2020). Damit tritt ein wesentlich vielschichtigerer Zusammenhang in den Blick, welcher das Verhältnis Mensch-Maschine kennzeichnet. Um diesen angemessen zu erfassen ist demnach eine stärker technikphilosophische Perspektive gefragt, die es einerseits erlaubt, ein solches Wechselverhältnis konkret zu erfassen und andererseits anschlussfähig ist für konkrete Rückschlüsse für demokratische Gesellschaften. 


\section{Eine technikphilosophische Kritik}

Die Angst um den Verlust individueller Freiheit bzw. Autonomie hat gerade in der politik- und sozialwissenschaftlichen Forschung Konjunktur: Sie tritt mit Blick auf unterschiedliche Etappen der Technikentwicklung in unterschiedlicher Art und Weise auf (Passoth 2008). Auch die Auseinandersetzung mit Algorithmen-basierten Systemen als gegenwärtiges Objekt der Angst reiht sich in diese Linie ein, werden diese doch als spezifische Gefährdungen individueller Freiheit und demokratischer Zusammenhänge wahrgenommen. Die Beziehung zwischen Menschen und digitalen Technologien lässt sich aber auch weniger deterministisch deuten. Denn eine Übertragung technischer Charakteristika auf gesellschaftliche Erscheinungsformen führt vor dem Hintergrund eines Gefahrendiskurses schnell in einen Zustand der permanenten Bedrohung für das moderne Individuum, der nur schwerlich aufgelöst werden kann. Dies wird besonders deutlich in der gerade beschriebenen Modellierung einer „,algorithmisch gesteuerten Gesellschaft“", in der „die Idee eines humanistischen Subjekts als primären Quells sozialer Ordnung zunehmend anachronistisch" erscheint (Block und Dickel 2020, S. 110). Forschungsfelder wie die Science \& Technology Studies oder die Technikphilosophie bieten dagegen bereits seit einigen Jahrzehnten Ansatzpunkte, um die Verknüpfung von Technik und Gesellschaft empirisch angemessener zu beschreiben und theoretisch gewinnbringend zu durchdenken, ohne einem starren Dualismus das Wort zu reden (Block und Dickel 2020; Hildebrandt und Rouvroy 2011; mit einem politikwissenschaftlichen Bezug siehe Berg et al. 2020 sowie Hofmann et al. 2019).

Solche Perspektiven legen das Augenmerk auf den Umstand, dass insbesondere moderne Gesellschaften nicht nur schon immer mit Technik vielfältig verflochten gewesen sind, sondern diese auch keine totalitäre Struktur neben der gesellschaftlich-individuellen Ebene ausbildet, demgegenüber also stets interdependent existiert (als systemtheoretisches Beispiel siehe Baecker 2019). Der Einsatz algorithmischer Systeme impliziert dann eben nicht die umfassende Transformation der Gesellschaft in eine automatisierte Feedbackmaschine (so etwa König 2018), sondern verweist vielmehr auf die Ausbildung technischer Assemblagen, die von den die jeweiligen Systeme auszeichnenden Logiken abweichende und dabei mitunter neue logischrationale Strukturen ausbilden können, diese kritisieren oder mit ihnen brechen das Verhältnis also wesentlich komplexer und diverser wird. In den Mittelpunkt rücken so die kontextabhängig wahrgenommenen Möglichkeitsstrukturen, Anreizlogiken sowie zielorientierte Akteur*innen, die in unterschiedlichen Netzwerken performativ ins Werk gesetzt werden (Berg et al. 2020). Menschen werden entsprechend nicht unmittelbar von der Maschine verobjektiviert, sie sind vielmehr in unterschiedlichen Handlungszusammenhängen miteinander verknüpft, die Prozesse der Subjektivierung und Objektivierung mitunter fließend.

Entsprechend kritisch gehen diese Ansätze mit Thesen einer objektivierten Subjektivität qua Technik um, die auch in der Diskussion um eine Algorithmisierung angeführt werden. Karin Knorr-Cetinas These einer Sozialität mit Objekten lässt sich dazu beispielhaft heranziehen (1998). In ihrem Beitrag diskutiert die Autorin die sozialwissenschaftliche These der Individualisierung als eine Entbettung aus den Strukturen menschlicher Gemeinschaft zugunsten einer vermeintlich entfremdeten 
Bindung an Objekte, etwa Instrumente oder Waren, die dann wahlweise in einer instrumentellen oder kommodifizierten Rationalität resultieren würde. Entlang KnorrCetinas These erscheinen Individuen wie Kollektive hingegen immer schon über Objekte vermittelt: Der Technik kommt hierbei eine bewusstseinsbildende Rolle zu, wobei jedoch gegenwärtig ,die ,Abstraktheit“ technologischer Produktion mit einem bestimmten kognitiven Stil in Zusammenhang gebracht" wird (85) - und dies in einem ausschließlichen Sinne: Wo Technik ist, finden wir eine durchrationalisierte Welt; wo verwaltet wird, ist der Mensch entfremdet.

Die in einem Dualismus verhaftete theoretische Übersetzung übersieht jedoch die „Kreolisierung des Sozialen“ (90 ff.) in modernen, von Wissenschaft und Technologie durchdrungenen Gesellschaften, deren Strukturen sich bereits seit Langem durch eine starke Vermischung mit expertischem und technologischem Wissen auszeichnen - und damit den im Diskurs um Algorithmen virulent werdenden Logiken formaler Rationalität. So gesprochen ignorieren solche einseitigen Definitionen ,die Verklammerung von Wissenskulturen und sozialen Strukturen“ (94). In ähnlicher Weise ließen sich auch algorithmische Systeme und KI, verstanden als „computergestützte Verfahren der Wissensproduktion“ (Ulbricht 2020, S. 255), als Aspekt einer Kreolisierung des Sozialen interpretieren, in welchem Menschen technologisch vielfältig vermittelt werden. Technologische Funktionslogiken, wie sie uns durch algorithmische Systeme gegenübertreten, müssen daher nicht zwingend in einem entfremdeten technologischen Bewusstsein münden, sondern bieten auch unterschiedliche Anknüpfungspunkte und Momente der Auseinandersetzung. Somit sind diese algorithmischen ,engines of order“ in komplementärer Weise zum Menschen daran beteiligt, politische Ordnungsbildung zu bewerkstelligen (Rieder 2020). Es geht also nicht um ein Unterwerfungs- oder Übernahmenarrativ, sondern um die Erkundung eines Wechselverhältnisses, vielleicht auch ihres Nebeneinander-Existierens. Mit Peter-Paul Verbeek ließe sich daher argumentieren:

We must give up the idea that we exercise a sovereign authority over technology and that we employ technologies merely as neutral means towards ends that have been autonomously determined. The truth is that we are profoundly technologically mediated beings. (Verbeek 2011, S. 30)

Ausgehend von der These der grundlegenden technologischen Mediatisiertheit des menschlichen Weltverhältnisses kann das Verhältnis des Menschen zur Technologie dann keines sein, das auf der simplen Dichotomie von Befreiung oder Unterdrückung, Autonomie oder Entfremdung beruht. Denn nicht nur die menschliche Existenz im Allgemeinen, auch politische Ordnungen und Gemeinschaften erschlieBen sich erst mittels des vielgestaltigen technologischen Netzes, das Individuen wie Gesellschaften durchzieht und umgibt - nicht unabhängig davon. Es ist somit anzunehmen, dass das Geflecht aus Individuen und digitalen Technologien ein auf Sinn gerichtetes, und somit auf Interpretation angewiesenes Verhältnis darstellt und deshalb gerade als ergebnisoffen verstanden werden muss. Zusammengefasst bedeutet dies dann gerade in Abgrenzung zu dualistischen Annahmen, dass Technologie auf einer sehr grundlegenden Weise Teil unserer Existenz war und seither ist. Gesellschaften demzufolge also ihre Existenz ebenso in Relation zu Technologie, nicht in der Abgrenzung, Unterwerfung oder Unterdrückung zu bzw. von ihnen entwerfen 
und gestalten - die Formen und Logiken, die solche Momente des Aufeinandertreffens annehmen sind somit wesentlich diffiziler (Verbeek 2011, S. 32). Folgt man Verbeeks Annahme, dass Menschen mediatisierte Wesen sind und geht mit der Annahme mit, dass dieses Charakteristikum auch auf moderne Demokratien zutrifft (so etwa Hofmann 2019), dann lohnt es sich zu fragen, ob und wie die Interaktion mit digitaler Technologie die Logik verändert, unter der sich politische Ordnungsbildung vollzieht und welche Auswirkungen dies auf demokratische Gesellschaften hat. Konkret rücken damit die Fragen in den Mittelpunkt, nach welcher Logik KI im konkreten Fall eigentlich operiert und wie sich dies zu einer demokratischen Logik relationiert.

\section{Demokratie als Auflösung formal-rationalistischer Begründungsbestrebungen}

In der Auseinandersetzung mit algorithmischen Verfahren wird die mit ihnen einhergehende Rationalisierung vor allem als Letztbegründungsanspruch algorithmischer Systeme verstanden - die ausgegebenen Kategorien, Empfehlungen und Entscheidungen stehen definitiv, ordnen oder entscheiden somit eine spezifische Situation eindeutig. So vertreten algorithmische Systeme als ontologisierend-deterministische Verfahren den Anspruch endgültigen Wissens, wobei dieses in einen direkten Konflikt mit den pluralistisch-reflexiven Logiken demokratischer Systeme tritt, die gerade die vielfältigen Begründungszusammenhänge und -möglichkeiten konkreter Sachverhalte betonen. Algorithmische Systeme führen damit Absolutheitsansprüche mit Verve erneut in das Feld des Politischen ein, welche sodann auf die nicht negierbare Veränderung und stete Möglichkeit zur Gestaltung demokratischer Gesellschaften prallen (Ulbricht 2020, S. 256). Sybille Krämer spricht deshalb schon im Zusammenhang mit dem Aufkommen von Computern von der Zerstörung der „symbolischen Differenz“ (1991, S. 8) durch formal-mechanische Systeme, wobei die Differenz ja gerade auf die Möglichkeiten unterschiedlicher Bedeutungsproduktion verweist. Dabei lässt sich Krämers Ausdruck in einsichtsvoller Weise zum Begriff der ,politischen Differenz“ (Marchart 2010) in Beziehung setzen, der insbesondere im Bereich der radikalen Demokratietheorie zu einem zentralen Angelpunkt der Auseinandersetzung um Politisierung und Depolitisierung avanciert ist (Bröckling und Feustel 2010).

In beiden Fällen steht die Differenz für eine produktive Beziehung zwischen den gesellschaftlichen bzw. institutionellen Arrangements und den sie fundierenden Normen und Werten. Während die symbolische Differenz Krämers grundlegend die der Mathematik eigenen Problemlösungsverfahren und ihre (Nicht-)Bezugnahme auf die mit Symbolen verbundenen Bedeutungsebenen im Feld der Mathematik herausarbeitet (Krämer 1991, S. 3 f.) und in einem weiteren Schritt auf die (moderne) Erkenntnistheorie überträgt, ist der Terminus der politischen Differenz vor allem in einer politisch-philosophischen Tradition verortet. Er betont innerhalb demokratischer Gesellschaften insbesondere die Möglichkeiten für Veränderung und Neugestaltung, die sich aus einer Abweichung zwischen der institutionellen Ausgestaltung und den sie fundierenden Werten und Normen ergeben (Marchart 2019, 
S. 572). Die reflexive Anerkennung von Ungewissheit lässt sich daher als kontingenter Horizont demokratischer Gesellschaften verstehen.

Eine Perspektivierung auf die politische Kontingenz moderner Demokratie bezeichnet aber gerade keinen ontologischen Status, sondern ein ,empirisch nachvollziehbar[es] Bewusstsein[s]- und Praxisverhältnis der Menschen in der Moderne, durch das ihnen ihre gesamte gesellschaftliche Lebenswelt latent kontingent und damit entscheidbar geworden ist“ (Greven 2010, S. 68). Die Auflösung der „Grundlagen aller Gewißheit“" (Lefort 1990, S. 296) verweist ja gerade auf den Umstand, dass in demokratischen Gesellschaften niemand mehr einen absoluten Anspruch auf Wahrheit zur Begründung politischer Handlungen und Ordnungsbildung argumentativ ins Feld führen kann. Erst der Umstand, dass für die Bürger*innen etwas entscheidbar geworden ist, erlaubt Politisierung.

In den Grundzügen geht die politikwissenschaftliche Kritik an Algorithmen-basierten Systemen jedoch über den Verweis auf die Unterminierung einer grundlegenden demokratischen Kontingenz und damit verbundene plurale Sinndimensionen nicht hinaus. Demokratien allein auf das Moment der Kontingenz zu reduzieren, würde allerdings zu kurz greifen und letztlich der Dynamik der Demokratie nicht ausreichend Rechnung tragen. Demokratische Politik geht nicht in einer für sich stehenden Feier der Pluralität auf, sondern ist eng geknüpft an eine gezielte Auseinandersetzung mit Gewissheitsansprüchen: Politik gilt immer (auch) als eine definitorische Ausgestaltung von Normen, Werten und Wahrheitsansprüchen. Institutionell lässt sich daher demokratische Politik als aktive Handlung, als eine iterative wie interaktive Praxis des Befragens verstehen (Flügel-Martinsen 2019, S. 462f., 2017), die sich Verfahren schafft, um diese Spannung aus Kontingenzbewusstsein und Wahrheitsansprüchen für eine politische Handlungsgemeinschaft handhabbar zu gestalten. Leforts Rede vom „leeren und unbesetzbaren Ort der Macht“ (Lefort 1990, S. 293) veranschaulicht dies. Denn einerseits verweist er auf eine immer aufs Neue bewusst gemachte Anerkennung von Ungewissheit, macht aber gleichzeitig die institutionelle Kanalisierung gesellschaftlicher Konflikte um die zumindest temporäre Setzung von Aussagen zur Voraussetzung demokratischer Politik deutlich.

Die Vermittlung der Ungewissheit erfolgt also gerade im repräsentativen Modus des Vorbringens von Vorschlägen einerseits und der interaktiven Auseinandersetzung mit ihnen andererseits. Damit erscheint politisches Handeln stets gerichtet auf sich formierende deterministische Strukturen und der damit verbundenen Setzung fester Prinzipien mit dem Ziel, diese erneut einer gesellschaftlichen Auseinandersetzung durch die Ausstellung von pluralistischen Deutungsangeboten qua Befragung und Kritik zuzuführen. Deutlich wird so ein sich wiederholender Prozess der Verständigung über ebenso temporäre wie prekäre Begründungen (Comtesse et al. 2019, S. 14). Ausgehend von Momenten der Pluralität, des Sinnüberschusses und NichtDeterminierbarkeit operiert politisches Handeln in einer steten Wechseldynamik der Verfestigung wie der Auflösung formal-rationalistischer Begründungsbestrebungen 
in Bezug auf soziale, ökonomische und kulturelle Sachverhalte. ${ }^{3}$ Entsprechend lassen sich diese Überlegungen auf den Punkt bringen, dass die ,eigentliche demokratische Freiheit der gesellschaftlichen Selbstkonstitution" sich gerade in einer demokratischen Praxis äußert, die iterativ die Fundamente der Macht, des Rechts und des Wissens in Frage stellt (Flügel-Martinsen 2015, S. 112). Damit ließe sich argumentieren, dass sich Demokratien gerade durch ein Ringen mit ontologisierenden Tendenzen auszeichnen, sich ihrer also in einem praktischen Vollzug annehmen etwa im Kontext einer profanen, nicht zwingend technologischen Verwaltung bzw. der Wissenschaft, die beide nicht nach demokratischen Logiken im engeren Sinne agieren und dennoch immer wieder politisiert werden. Demokratien können somit als geübt im Umgang mit Prozessen der epistemologischen Schließung verstanden werden - vorausgesetzt, man sieht algorithmische Verfahren sowie den durch die generierten Output als repräsentativen Vorschlag einer Weltdarstellung, über den es zu diskutieren gilt - nicht als naturalisierten Fakt.

Was bedeutet dies nun für die Einbettung von KI und Algorithmisierung in politische Kontexte demokratischer Systeme? Eine demokratietheoretische Auseinandersetzung kann nicht im Beklagen der Unterminierung ihrer pluralen Sinnbezüge, also in der Annahme, dass der Einsatz von KI oder algorithmischer Systeme im Politischen zu einer De-Politisierung führen würde, stehen bleiben, sondern muss im Sprechen über Kontingenz auch den Modus der demokratischen Auseinandersetzung mit postulierten Gewissheiten betrachten und auf epistemische Praktiken algorithmischer Systeme übertragen. In einem demokratischen Interaktionszusammenhang muss entsprechend das Aufscheinen einer vereindeutigenden Logik von KI nicht als die Verunmöglichung von Ungewissheit gelesen werden. So würde es sich nicht um eine Auflösung demokratischer durch technologische Logiken handeln, sondern vielmehr als eine stete, jedoch nur punktuelle Neutralisierung von Ungewissheit erscheinen (Esposito 2007, S. 4). Eine solche punktuelle Neutralisierung wiederum ist in einen größeren Handlungszusammenhang eingebunden und kann stets hinterfragt werden, wodurch wieder neue Ungewissheiten produziert werden können, indem die vorgestellten Sachverhältnisse in Zweifel gezogen werden. Dies verweist auf einen wesentlichen Aspekt mediatisierter Demokratie: Sie ist ein vermittelter und vermittelnder Prozess, der gerade geprägt ist durch Prozesse der Befragung von Repräsentant*innen und Beauftragten, seien sie nun politischer, kultureller, wirtschaftlicher oder wissenschaftlicher Natur, wobei insbesondere letztere auch durch technologische Verfahren unterstützt sein können. Entsprechend wird häufig der Aspekt unterschlagen, dass in politischen Debatten grundsätzlich ein Wettstreit unterschiedlicher epistemischer Modi stattfindet. Es geht demnach um ,a nuanced understanding of the epistemic contests [...] that populate public life, as well as the complex array of social and institutional processes within which

\footnotetext{
3 So findet sich etwa eine Kritik an der rationalistischen Auflösung von Entscheidungsproblemen nicht erst seit der Gewahrwerdung der mechanischen Unterlegung von Kommunikationsvorgängen, sondern im Bereich der Demokratietheorie auch in Bezug auf die rationale Auflösung von Zielkonflikten - wie dies etwa deliberativen Entwürfen der Demokratie vorgeworfen wird (zur Kritik an einer kognitivistischen Interpretation der Demokratie etwa Thaa 2011; Urbinati 2010).
} 
these contests take place and policy-relevant facts and ideas are formed, validated, critiqued, disseminated, and discarded.“ (Miller 2008).

Die Einbettung von KI in politische Kontexte erscheint daher als Fortführung einer Praxis des Regierens, die sowohl in der Gubernative wie auch der Administration komplexitätsreduzierend und instrumentell agiert, ohne dass dadurch der politische Charakter grundsätzlich negiert würde. Zumal insbesondere aus demokratietheoretischer Sicht die Annahme einer absoluten Herrschaft wenig überzeugend scheint das gilt für institutionelle Settings und muss auch auf technisierte Umgebungen übertragen werden. Es muss also wesentlich präziser danach gefragt werden, ob und in welchem Ausmaß in Umgebungen algorithmischer Systeme Formen der Kritik und Befragung ermöglicht werden oder nicht, und ob Algorithmen darauf Einfluss haben, was und wie kritisiert und befragt werden kann (Rieder und Hofmann 2020).

Dabei wird deutlich, dass auch Verfahren der Algorithmisierung im Allgemeinen oder KI im Speziellen Handlungsräume der Befragung, der Kritik und der Politisierung zulassen - und das auf vielfältigen Ebenen des zivilgesellschaftlichen Engagements oder des Protestes. Ausgearbeitet hat das jüngst etwa Lena Ulbricht. Sie untersucht Formen der Politisierung algorithmischer Systeme, die sich auch auf KI übertragen lassen (Ulbricht 2020, S. 265). Wo sich einerseits eine Depolitisierung durch Algorithmen verzeichnen lässt - durch Komplexitätsreduktion und Formalisierung -, lässt sich darüber hinaus im Umgang mit diesen Verfahren auch eine RePolitisierung feststellen. Dabei zeichnet sich eine Politisierung vor allem dadurch aus, dass die über algorithmische Systeme propagierten sozialen wie politischen Normen hinterfragt und kritisch beleuchtet werden - dies nicht selten mit dem Hinweis auf Diskriminierung, Benachteiligung und Ungerechtigkeiten (Benjamin 2019). Ein prägendes Beispiel hierfür sind etwa die „Fuck the Algorithm“-Proteste in Großbritannien, die sich konkret gegen einen Algorithmus richteten: Durch die Corona-bedingte Schließung der Schulen stellte sich die Frage nach einer angemessenen Beurteilung schulischer Leistungen aufgrund der langen Schließzeiten und ausgefallener Prüfungen. Die Idee hinter dem Einsatz und der Programmierung des Systems war es, die Benotung der Schüler*innen an den vorangegangenen Jahren $\mathrm{zu}$ orientieren, also ein Muster zu erarbeiten, dass sich in die Zukunft projizieren ließe, ohne dabei individuelle Personen zu bevorzugen und stattdessen eine gewisse Kontinuität sicher zu stellen. Es stellte sich jedoch heraus, dass die Schüler*innen wesentlich schlechter benotet wurden als in den Jahren zuvor - dies korrelierte mit der geographische Lage der Schule. Es zeigte sich, dass neben den konkreten schulischen Noten auch die landesweite Vergabe von Noten mit einbezogen wurde und so zu einer spezifischen sozialen Verzerrung führte und auf die individuellen Schüler*innen übertragen wurde. Befand sich die Schule in einer Region, in der der Notendurchschnitt höher lag, erhielten auch die Schüler*innen im Schnitt eine bessere Note. Der Algorithmus war so also gerade nicht in der Lage, konkrete soziale wie ökonomische Parameter angemessen mit einzubeziehen, die konkrete klassenspezifische Lagen in einem größeren Kontext ausmachten und so bereits marginalisierte Schüler*innen härter traf (AlgorithmWatch 2020, S. 280).

Zwar bestand die Möglichkeit, Beschwerde gegen die Benotung einzureichen, doch wurde darüber hinaus vor allem öffentlich auf die den Algorithmen inhärenten Logiken aufmerksam gemacht und das System als solches diskreditiert. Die Risi- 
ken der Nicht-Differenzierung und falschen Verallgemeinerung durch algorithmische Systeme führte zu direkten gesellschaftlichen Reaktionen, die sich in Protesten der betroffenen Jugendlichen äußerten. Es zeigt sich also, dass gerade die algorithmische Logik der Vereindeutigung, Verobjektivierung und Nicht-Differenziertheit zum Gegenstand einer öffentlichen Kritik werden kann und damit neue Sinnhorizonte eröffnete, die gerade die algorithmische Logik in ihrer numerisch-deterministischen Form als ungeeignet herausstellen. Ermöglicht und angeleitet wurde die Kritik, wie bereits Ulbricht in Auseinandersetzung mit dem Einsatz algorithmischer Systeme durch staatliche Institutionen zeigte, vor allem über die Anführung von Werten der Pluralität, Ambiguität und dem Anzeigen einer falschen Gleichbehandlung, die wiederum in Ungleichheit mündet (Ulbricht 2020, S. 270).

Algorithmische Systeme bzw. die sie anleitenden Logiken sind politisierbar und ermöglichen somit Momente der Re-Politisierung (ebd.: $268 \mathrm{f}$.). Ulbricht etwa konnte zeigen, dass gerade beim staatlichen Einsatz algorithmischer Systeme eine Kritik etwa durch zivilgesellschaftliche Akteur*innen oder Medien erfolgreich sein kann, wobei sich diese Entwicklung auch im privatwirtschaftlichen Bereich beobachten lässt (ebd.: 261-266). Darüber hinaus scheint der Bereich der Produktplatzierung bzw. individueller Werbung in den Blick der kritischen Auseinandersetzung zu rücken vor allem in Wahlkampfzeiten, wenn über gezielte Parteienwerbung nach Personengruppen gesprochen wird und Aspekte der digitalen Wähler*innenbeeinflussung betont werden. Stichworte bilden hier Begriffe wie ,,voter targeting“ oder auch ,microtargeting“, wobei es darum geht, auf einer breiten Datenbasis die Präferenzen und Wünsche der Wähler*innen zu ermitteln und auf Basis dessen Wahlkämpfe zu führen und Wahlen zu gewinnen (Bennett und Lyon 2019). Auch Prozesse der digitalen Überwachung rücken zunehmend ins Visier zivilgesellschaftlicher Kritik (Kazansky und Milan 2021). Entgegen der Metapher algorithmischer Systeme als „black boxes“ (Pasquale 2015) entzieht sich ihre Logik nicht völlig einer demokratischen Auseinandersetzung, da hier gerade die gesellschaftliche Einbettung zum Ziel und Mittel der Auseinandersetzung mit algorithmischen Logiken avanciert, welche praktisch befragt und einer Kritik unterzogen werden können.

Demokratietheoretisch scheint es daher geboten, sich stärker systematisch einer kritischen und techniksensiblen Befragung von KI oder algorithmischen Systemen zu widmen, um angemessen auf die umfassende Technisierung politischer Zusammenhänge in der digitalen Konstellation reagieren zu können. Folgt man der These der Rekonstruktion von KI als symbolischem Verfahren, das in die Produktion von Bedeutung und Wirklichkeit interveniert, bietet die kontingenztheoretische Bedeutung demokratischer Praktiken einen möglichen Ansatzpunkt. Es ließe sich etwa fragen, ob algorithmische Systeme die Grenzen der gesellschaftlichen Kontingenz selbst verschieben, was in Verbindung mit algorithmischen Logiken überhaupt in Frage gestellt werden kann oder wie etwa Modi der Kritik sich selbst in Auseinandersetzung mit digitalen Technologien verändern (Hörl et al. 2021b). Damit rückt der Blick einerseits weg von überzeichnenden und pauschalisierenden Tendenzen hin zu konkreten Fragen der praktischen und institutionellen Einbettung von Algorithmenbasierten Verfahren in Prozesse gesellschaftlicher oder politischer Vermittlung. Andererseits geht mit dieser perspektivischen Verschiebung einher, dass digitale und 
technische Phänomene prägnanter im Feld politiktheoretischer Fragen erscheinen und somit angemessen der Diskussion zugeführt werden können.

Danksagung Für hilfreiche Anmerkungen und kritische Rückfragen möchte ich mich bei Michael Klipphahn, Richard Groß und Lukas Nehlsen sowie bei Prof. Dr. Sabine Müller-Mall und dem gesamten Lehrstuhl-Team bedanken. Dank gilt darüber hinaus den Herausgebern des Heftes Sebastian Berg, Daniel Staemmler und Thorsten Thiel für kritische Anmerkungen sowie den zwei anonymen Gutachter*innen für wertvolle Anregungen und Kommentare.

Funding Open Access funding enabled and organized by Projekt DEAL.

Open Access Dieser Artikel wird unter der Creative Commons Namensnennung 4.0 International Lizenz veröffentlicht, welche die Nutzung, Vervielfältigung, Bearbeitung, Verbreitung und Wiedergabe in jeglichem Medium und Format erlaubt, sofern Sie den/die ursprünglichen Autor(en) und die Quelle ordnungsgemäß nennen, einen Link zur Creative Commons Lizenz beifügen und angeben, ob Änderungen vorgenommen wurden.

Die in diesem Artikel enthaltenen Bilder und sonstiges Drittmaterial unterliegen ebenfalls der genannten Creative Commons Lizenz, sofern sich aus der Abbildungslegende nichts anderes ergibt. Sofern das betreffende Material nicht unter der genannten Creative Commons Lizenz steht und die betreffende Handlung nicht nach gesetzlichen Vorschriften erlaubt ist, ist für die oben aufgeführten Weiterverwendungen des Materials die Einwilligung des jeweiligen Rechteinhabers einzuholen.

Weitere Details zur Lizenz entnehmen Sie bitte der Lizenzinformation auf http://creativecommons.org/ licenses/by/4.0/deed.de.

\section{Literatur}

AlgorithmWatch. 2020. Report Automating Society 2020. : AlgorithmWatch, Bertelsmann.

Amoore, Louise. 2020. Cloud ethics. Algorithms and the attributes of ourselves and others. Durham: Duke University Press.

Araujo, Theo, Natali Helberger, Sanne Kruikemeier, und Claes H. de Vreese. 2020. In AI we trust? Perceptions about automated decision-making by artificial intelligence. AI \& SOCIETY 35:611-623. https:// doi.org/10.1007/s00146-019-00931-w.

Bächle, Thomas Christian. 2016. Digitales Wissen, Daten und Überwachung zur Einführung. Hamburg: Junius.

Baecker, Dirk. 2019. Intelligenz, künstlich und komplex. Leipzig: Merve.

Becker, Carlos, und Sandra Seubert. 2020. Die Selbstgefährdung der Autonomie. Eckpunkte einer Kritischen Theorie der Privatheit im digitalen Zeitalter. In Digitale Transformationen der Öffentlichkeit, Hrsg. Jan-Philipp Kruse, Sabine Müller-Mall, 229-261. Weilerswist: Velbrück Wissenschaft.

Benjamin, Ruha. 2019. Race after technology: abolitionist tools for the new Jim code. Medford: Polity.

Bennett, Colin J., und David Lyon. 2019. Data-driven elections. Implications and challenges for democratic societies. Internet Policy Review 8:1-16. https://doi.org/10.14763/2019.4.1433.

Berg, Sebastian, Niklas Rakowski, und Thorsten Thiel. 2020. Die digitale Konstellation. Eine Positionsbestimmung. Zeitschrift für Politikwissenschaft 30:171-191. https://doi.org/10.1007/s41358-02000207-6.

Block, Katharina, und Sascha Dickel. 2020. Jenseits der Autonomie. Die De/Problematisierung des Subjekts in Zeiten der Digitalisierung. Behementh A Journal on Civilisation 13:109-131. https://doi.org/ 10.6094/BEHEMOTH.2020.13.1.1040.

Boden, Margaret. 1990. Introdction. In The philosophy of artificial intelligence, Hrsg. Margaret A. Boden, 1-21. Oxford, New York: Oxford University Press.

Bröckling, Ulrich, und Robert Feustel (Hrsg.). 2010. Das Politische denken. Bielefeld: transcript.

Bucher, Taina. 2018. If...then. Algorithmic power and politics. New York: Oxford University Press.

Comtesse, Dagmar, Oliver Flügel-Martinsen, Franziska Martinsen, und Martin Nonhoff. 2019. Einleitung. In Radikale Demokratietheorie: Ein Handbuch, Hrsg. Dagmar Comtesse, Oliver Flügel-Martinsen, Franziska Martinsen, und Martin Nonhoff, 11-20. Berlin: Suhrkamp. 
Coy, Wolfgang. 1993. Reduziertes Denken. Informatik in der Tradition des formalistischen Forschungsprogramms. In Informatik und Philosophie, Hrsg. Peter Schefe, Margaret A. Boden, 31-52. Mannheim: B.I. Wissenschaftsverlag.

Domingos, Pedro. 2015. The master algorithm. How the quest for the ultimate learning machine will remake our world. London: Penguin Books.

Egbert, Simon. 2020. Datafizierte Polizeiarbeit. (Wissens-)Praktische Implikationen und rechtliche Herausforderungen. In Polizeiarbeit zwischen Praxishandeln und Rechtsordnung. Empirische Polizeiforschungen zur polizeipraktischen Ausgestaltung des Rechts, Hrsg. Daniela Hunold, Andreas Ruch, 77-100. Wiesbaden: Springer VS.

Ernst, Christoph, Irina Kaldrack, Jens Schröter, und Andreas Sudmann. 2019. Künstliche Intelligenzen. Einleitung in den Schwerpunkt. Zeitschrift für Medienwissenschaft 21:10-19. https://doi.org/10. 25969/mediarep/12616.

Ertel, Wolfgang. 2016. Grundkurs Künstliche Intelligenz: Eine praxisorientierte Einführung. Wiesbaden: Springer.

Esposito, Elena. 2007. Die Fiktion der wahrscheinlichen Realität. Frankfurt am Main: Suhrkamp.

Flügel-Martinsen, Oliver. 2015. Das Abenteuer der Demokratie. Ungewissheit als demokratietheoretische Herausforderung. In Ordnungsbildung und Entgrenzung, Hrsg. Renate Martinsen, 105-119. Wiesbaden: Springer.

Flügel-Martinsen, Oliver. 2017. Befragungen des Politischen. Subjektkonstitution - Gesellschaftsordnung - Radikale Demokratie. Wiesbaden: Springer.

Flügel-Martinsen, Oliver. 2019. Befragung, negative Kritik, Kontingenz. Konturen einer kritischen Theorie des Poltischen. In Kritische Theorie der Politik, Hrsg. Ulf Bohmann, Paul Sörensen, 450-469. Berlin: Suhrkamp.

Gillespie, Tarleton. 2018. Platforms are not intermediaries. Georgetown Law Technology Review 2:198-216.

Glymour, Clark N., Kenneth M. Ford, und Patrick J. Hayes. 2006. The prehistory of android epistemology. In Android epistemology, Hrsg. Kenneth M. Ford, Clark N. Glymour, und Patrick J. Hayes, 3-23. Cambridge: MIT Press.

Greven, Michael Th. 2010. Verschwindet das Politische in der politischen Gesellschaft? Über Strategien der Kontingenzverleugnung. In Das Politische und die Politik, Hrsg. Thomas Bedorf, Kurt Röttgers, 68-101. Berlin: Suhrkamp.

Haugeland, John. 1993. Artificial intelligence: the very idea. Cambridge: MIT Press.

Heintz, Bettina. 1993. Die Herrschaft der Regel: zur Grundlagengeschichte des Computers. Frankfurt/New York: Campus.

Hildebrandt, Mireille, und Antoinette Rouvroy (Hrsg.). 2011. Law, human agency and autonomic computing. The philosophy of law meets the philosophy of technology. New York: Routledge.

Hofmann, Jeanette. 2019. Mediatisierte Demokratie in Zeiten der Digitalisierung. Eine Forschungsperspektive. In Politik in der digitalen Gesellschaft, Hrsg. Jeanette Hofmann, Norbert Kersting, Claudia Ritzi, und Wolf J. Schünemann, 27-46. Bielefeld: transcript.

Hofmann, Jeanette, Norbert Kersting, Wolf J. Schünemann, und Claudia Ritzi (Hrsg.). 2019. Politik in der digitalen Gesellschaft. Zentrale Problemfelder und Forschungsperspektiven. Bielefeld: transcript.

Hörl, Erich, Nelly Y. Pinkrah, und Lotte Warnsholdt. 2021a. Introduction. In Critique and the digital, Hrsg. Erich Hörl, Nelly Y. Pinkrah, und Lotte Warnsholdt, 7-24. Zürich: Diaphanes.

Hörl, Erich, Nelly Y. Pinkrah, und Lotte Warnsholdt (Hrsg.). 2021b. Critique and the digital. Zürich: Diaphanes.

Houben, Daniel. 2018. Instrumentelle Vernunft in der Datengesellschaft: Zur Relevanz der Kritischen Theorie für das Verständnis der Datafizierung des Sozialen. In Digitale Transformation im Diskurs: Kritische Perspektiven auf Entwicklungen und Tendenzen im Zeitalter des Digitalen, Hrsg. Christian Leineweber, Claudia de Witt, 197-220. Hagen: deposit_hagen. https://doi.org/10.18445/20180315092808-0.

Isaac, Alistar M.C. 2019. Computational thought from descartes to lovelace. In The Routledge handbook of the computational mind, Hrsg. Mark Sprevak, 9-22. New York: Routledge.

Just, Natascha, und Michael Latzer. 2017. Governance by algorithms. Reality construction by algorithmic selection on the Internet. Media, Culture \& Society 39:238-258. https://doi.org/10.1177/ 0163443716643157.

Katzenbach, Christian, und Lena Ulbricht. 2019. Algorithmic governance. Internet Policy Review 8:1-18. https://doi.org/10.14763/2019.4.1424.

Kazansky, Becky, und Stefania Milan. 2021. "Bodies not templates". Contesting dominant algorithmic imaginaries. New Media \& Society 23:363-381. https://doi.org/10.1177/1461444820929316. 
Kitchin, Rob. 2017. Thinking critically about and researching algorithms. Information, Communication \& Society 20:14-29. https://doi.org/10.1080/1369118X.2016.1154087.

Knorr-Cetina, Karin. 1998. Sozialität mit Objekten. Soziale Beziehungen in posttraditionalen Wissensgesellschaften. In Technik und Sozialtheorie, Hrsg. Werner Rammert, 83-120. Frankfurt am Main: Campus.

König, Pascal D. 2018. Algorithmen und die Verwaltung sozialer Komplexität: Zur Neukonfigurierung der Idee der Selbstregierung des Volkes. Zeitschrift für Politikwissenschaft 28:289-312. https://doi.org/ 10.1007/s41358-018-0162-z.

König, Pascal D. 2019. Die digitale Versuchung. Wie digitale Technologien die politischen Fundamente freiheitlicher Gesellschaften herausfordern. Politische Vierteljahresschrift 60:441-459. https://doi. org/10.1007/s11615-019-00171-z.

Krämer, Sybille. 1988. Symbolische Maschinen: die Idee der Formalisierung in geschichtlichem Abriss. Darmstadt: Wissenschaftliche Buchgesellschaft.

Krämer, Sybille. 1991. Berechenbare Vernunft. Kalkül und Rationalismus im 17. Jahrhundert. Berlin, New York: De Gruyter.

Lefort, Claude. 1990. Die Frage der Demokratie. In Autonome Gesellschaft und libertäre Demokratie, Hrsg. Ulrich Rödel, 281-297. Frankfurt am Main: Suhrkamp.

Lopez, Paola. 2021. Artificial Intelligence und die normative Kraft des Faktischen. Merkur 75:42-52.

Mackenzie, Adrian. 2017. Machine learners. Archaeology of a data practice. Cambridge: MIT Press.

Marchart, Oliver. 2010. Die politische Differenz. Berlin: Suhrkamp.

Marchart, Oliver. 2019. Kontingenz/Grundlosigkeit. In Radikale Demokratietheorie. Ein Handbuch, Hrsg. Dagmar Comtesse, Oliver Flügel-Martinsen, Franziska Martinsen, und Martin Nonhoff, 572-575. Berlin: Suhrkamp.

McCarthy, John. 2006. The philosophy of AI and the AI of philosophy. Stanford university, working paper. http://jmc.stanford.edu/articles/aiphil2/aiphil2.pdf. Zugegriffen: 22.07.2021.

McCarthy, John, Marvin L. Minsky, Nathaniel Rochester, und Claude E. Shannon. 1955. A proposal for the Dartmouth Summer Research Project on Artificial Intelligence. AI Magazin 27:12-14.

Mersch, Dieter. 2019. Ideen zu einer Kritik ,algorithmischer“ Rationalität. Deutsche Zeitschrift für Philosophie 67:851-873. https://doi.org/10.1515/dzph-2019-0062.

Miller, Clark A. 2008. Civic Epistemologies. Constituting Knowledge and Order in Political Communities. In Sociology Compass 2/6:1896-1919. https://doi.org/10.1111/j.1751-9020.2008.00175.x.

Mühlhoff, Rainer. 2020. Human-aided artificial intelligence: Or, how to run large computations in human brains? Toward a media sociology of machine learning. New Media \& Society 22:1868-1884. https:// doi.org/10.1177/1461444819885334.

Müller-Mall, Sabine. 2020. Freiheit und Kalkül. Die Politik der Algorithmen. Stuttgart: Reclam.

Pasquale, Frank. 2015. The black box society. The secret algorithms that control money and information. Cambridge: Harvard University Press.

Passoth, Jan-Hendrik. 2008. Technik und Gesellschaft: sozialwissenschaftliche Techniktheorien und die Transformationen der Moderne. Wiesbaden: VS.

Rieder, Bernhard. 2020. Engines of order: a Mechanology of algorithmic techniques. Amsterdam: Amsterdam University Press.

Rieder, Bernhard, und Jeanette Hofmann. 2020. Towards platform observability. Internet Policy Review 9:1-28. https://doi.org/10.14763/2020.4.1535.

Roberge, Jonathan, und Robert Seyfert. 2017. Was sind Algorithmuskulturen? In Algorithmuskulturen, Hrsg. Robert Seyfert, Jonathan Roberge, 7-40. Bielefeld: transcipt.

Rouvroy, Antoinette. 2011. Technology, virtuality and utopia. Governmentality in an age of autonomic computing. In Law, human agency and autonomic computing, Hrsg. Mireille Hildebrandt, Antoinette Rouvroy, 119-140. New York: Routledge.

Russell, Stuart J., und Peter Norvig. 2012. Künstliche Intelligenz: Ein moderner Ansatz. München, Harlow, Amsterdam: Pearson.

Seyfert, Robert, und Jonathan Roberge (Hrsg.). 2017. Algorithmuskulturen. Über die rechnerische Konstruktion der Wirklichkeit. Bielefeld: transcript.

Sprenger, Florian. 2019. Epistemologien des Umgebens. Zur Geschichte, Ökologie und Biopolitik künstlicher environments. Bielefeld: transcript.

Sudmann, Andreas. 2018. Zur Einleitung. Medien, Infrastrukturen und Technologien des maschinellen Lernens. In Machine Learning. Medien, Infrastrukturen und Technologien der Künstlichen Intelligenz, Hrsg. Christoph Engemann, Andreas Sudmann, 9-36. Bielefeld: transcript.

Sudmann, Andreas (Hrsg.). 2019. The democratization of artificial intelligence. Net politics in the era of learning algorithms. Bielefeld: transcript. 
Thaa, Winfried. 2011. Die Krise politischer Repräsentation. Eine Folge der Auflösung gesellschaftlicher Großgruppen und sozio-kultureller Milieus? In Krise und Reform politischer Repräsentation, Hrsg. Markus Linden, Winfried Thaa, 125-147. Baden-Baden: Nomos.

Thiel, Thorsten. 2020. Demokratie in der digitalen Konstellation. In Einführung in die Politische Theorie. Grundlagen-Methoden-Debatten, Hrsg. Gisela Riescher, Beate Rosenzweig, und Anna Meine, 331-349. Stuttgart: Kohlhammer.

Turing, Alan M. 1990. Computing machinery and intelligence. In The philosophy of artificial intelligence, Hrsg. Margaret A. Boden, 40-66. Oxford, New York: Oxford University Press.

Ulbricht, Lena. 2020. Algorithmen und Politisierung. Leviathan 48:253-278. https://doi.org/10.5771/ 9783748904076-253.

Ulbricht, Lena, Sebastian Haunss, Jeanette Hofmann, Ulrike Klinger, Jan-Hendrik Passoth, Christian Pentzold, Ingrid Schneider, Holger Straßheim, und Jan-Peter Voß. 2018. Dimensionen von Big Data: Eine politikwissenschaftliche Systematisierung. In Big Data und Gesellschaft: Eine multidisziplinäre Annäherung, Hrsg. Barbara Kolany-Raiser, Reinhard Heil, Carsten Orwat, und Thomas Hoeren, 151-231. Wiesbaden: Springer VS.

Unger, Sebastian, und Antje Ungern-Sternberger (Hrsg.). 2019. Demokratie und künstliche Intelligenz. Tübingen: Mohr Siebeck.

Ungern-Sternberger, Antje. 2019. Demokratische Meinungsbildung und künstliche Intelligenz. In Demokratie und künstliche Intelligenz, Hrsg. Sebastian Unger, Antje Ungern-Sternberger, 3-31. Tübingen: Mohr Siebeck.

Urbinati, Nadia. 2010. Unpolitical democracy. Political Theory 38:65-92. https://doi.org/10.1177/ 0090591709348188.

Verbeek, Peter-Paul. 2011. Subject to technology. On automatic computing and human autonomy. In Law, human agency, and autonomic computing: the philosophy of law meets the philosophy of technology, Hrsg. Mireille Hildebrandt, Antoinette Rouvroy, 27-45. New York: Routledge.

Winograd, Terry, und Fernando Flores. 1989. Erkenntnis, Maschinen, Verstehen. Zur Neugestaltung von Computersystemen. Berlin: Rotbuch.

Yeung, Karen. 2018. Algorithmic regulation: a critical interrogation: algorithmic regulation. Regulation \& Governance 12:505-523. https://doi.org/10.1111/rego.12158. 